\title{
Training courses on emergency preparedness, response and recovery: theory, practice and application of newly developed tools
}

\author{
C. Turcanu ${ }^{1}$, P. Crouäil ${ }^{2}$, T. Duranova ${ }^{3}$, J. Camps ${ }^{1}$, T. Schneider ${ }^{2}$ and W. Raskob ${ }^{4}$ \\ ${ }^{1}$ SCK•CEN - Belgian Nuclear Research Centre, Mol, Belgium. \\ ${ }^{2}$ CEPN - Centre d'étude sur l'Evaluation de la Protection dans le domaine Nucléaire, Fontenay-aux-Roses, France. \\ 3 VUJE, Inc, Trnava, Slovakia. \\ ${ }^{4}$ KIT - Karlsruhe Institute of Technology, Eggenstein-Leopoldshafen, Germany.
}

\begin{abstract}
Education and training remains an essential factor ensuring the creation and maintenance of critical capacities in the field of emergency preparedness, response and recovery. Within the FP7 PREPARE project, several training activities were organised: (i) basic courses for key players, addressing the early and intermediate phases of emergency preparedness and response as well as the long-term management of contaminated territories; and (ii) course on scientific methods and tools for information collection and exchange, namely the PREPARE analytical platform.
\end{abstract}

Keywords: training / emergency preparedness and response / recovery / analytical platform

\section{Introduction}

Planning and preparedness are key factors for efficient emergency management and recovery. This requires continuous training for technical and radiological advisors, staff responsible for the overall emergency organisation and policy, such as civil protection officers and environmental protection officers, either entering the domain or being interested in refreshing the basics and getting acquainted with latest developments in the field.

For recovery issues, education and training $(\mathrm{E} \& \mathrm{~T})$ plays a different role, being mainly devoted to develop the awareness, among radiation protection experts and authorities, of the specificities of managing long-lasting exposure situations, taking into account the concerns and worries of affected people.

Off-site nuclear emergency management has seen major advances in recent years. This notably includes development of improved guidance on the protection of population and the environment in the case of a nuclear accident or radiological emergency, enhanced decision support tools, updated information and data exchange and increasing focus on stakeholder participation, notably for the post-accident management.

The aftermath of the nuclear accident in Japan following the tragic earthquake and tsunami has emphasised again the need to thoroughly prepare all aspects of nuclear emergency planning and response and has reinforced the need for the development of the radiation protection culture and participation of stakeholders in the decision-making processes in the recovery phase.

In the framework of the FP7 project PREPARE, several training activities were organised:
- Basic training of key players in the field of nuclear and radiological emergency and post-accident management by the organisation of two basic courses:

- training course on preparedness and response for nuclear and radiological emergencies;

- training course on late phase nuclear accident preparedness and management.

- Training related to the scientific methods and tools for information collection and exchange developed or updated in the PREPARE project:

- training course on the PREPARE analytical platform (AP).

The scope of these training activities as well as the main conclusions regarding future needs for $\mathrm{E} \& \mathrm{~T}$ in the field of nuclear emergency preparedness, response and recovery are summarised in the following sections.

\section{Basic courses for key players}

Making informed and effective decisions in nuclear and radiological emergencies and recoveries requires not only a good understanding of the theoretical background of radiological evaluations, the principles of intervention, the planning and organisation in off-site emergency response and the multiple factors influencing decisions, but also on-the-field immersion (e.g. in an affected territory) and face-to-face testimonies from people who have really experienced (or are still facing) the management of accidental or post-accidental exposure situations. 
In the context of the FP7 project PREPARE, two complementary basic courses for key players were organised addressing the training needs of key management players along the entire cycle of nuclear/radiological emergency and recovery management. The first course focused on the early to intermediate phases after a nuclear/radiological accident and took place in Mol (Belgium), whereas the second addressed the long-term management of contaminated territories and took place in Gomel (Belarus).

The courses were organised in collaboration with main European and International emergency management actors 5 and the European platform NERIS (Preparedness for Nuclear and Radiological Emergency Response and Recovery, www.eu-neris.net) and the Research Institute of Radiology in Belarus, with the participation of Belarusian, Norwegian and Japanese experts, involved in the management of the long-term consequences of the Chernobyl and Fukushima accidents.

The objectives of the training courses were to provide the participants with theoretical background and practical knowledge required to make informed and effective decisions and to develop participatory processes involving local stakeholders in nuclear and radiological emergencies and recoveries:

- radiological evaluations;

- principles of intervention;

- planning and organisation of off-site emergency response;

- factors influencing decisions;

- principles for the management of existing exposure situations for the recovery phase;

- on-the-field immersion (e.g. in an affected territory);

- face-to-face testimonies from people experiencing the management of accidental or post-accidental exposure situations; and

- role of the radiation protection culture for engaging stakeholders in the decision-making processes.

Previous to the FP7 PREPARE project, the basic courses for key players have been organised in various countries, in the framework of other European projects. More than 500 specialists, mainly from European countries, attended these courses. The audience extended within the PREPARE project to include nuclear safety and radiation protection authorities, research institutes, emergency response organisations, first responders, CBRN officers and students (University of Hiroshima - PHOENIX Programme).

The content of the courses has not only been harmonised within PREPARE but also revised drawing on feedback from past experience, when such courses have been carried out either in the framework of NERIS or other international projects, and the new findings from PREPARE and the feedback from Fukushima accident management and recovery.

The comments received from the participants highlight that increasing attention is required for:

5 BfS (Germany), CEPN (France), CRMHE (Belarus), EOC (Switzerland), EPA (Ireland), European Commission, IAEA, ICRP, IRSN (France), JRC Ispra (Italy), KIT (Germany), NRPA (Norway), PHE (UK), RIR (Belarus), Tokyo Healthcare University, Tokyo University and Minamisoma Hospital (Japan).
- basic notions of radiation protection;

- radiological incidents and accidents, e.g. transport accidents or events related to the malevolent use of ionising radiation;

- practical aspects of intervention, e.g. protection of intervention personnel, zoning of affected areas, timedependent effectiveness of protective actions;

- communication, social and psychological impact for emergency management;

- health surveillance including long-term issues in the perspective of contributing to the improvement of the living conditions of affected populations;

- stakeholder engagement processes and development of the radiation protection in the recovery efforts; and

- lessons learned from past nuclear or radiological accidents.

In general, there is a need to address, in a balanced way, the theoretical and practical aspects of emergency and recovery management. This was realised in the basic courses for key players through table-top exercises, presentation or demonstrations of measurement equipment and laboratories or decontamination facilities, site visits, and discussions and debates with people involved in emergency preparedness or 'on the spot' post-accident rehabilitation strategies.

The basic courses received very good evaluations from the participants, which pointed out the continuous need for training in emergency preparedness, response and recovery. Future training programmes should cover more extensively radiological events and might be designed in flexible course formats for specific targeted audiences, depending on background knowledge and the specific emergency management or recovery tasks they are responsible for.

\section{Course on specialised tools: the PREPARE analytical platform}

The training course on the PREPARE 'AP - scientific methods and tools for information collection and exchange' was organised by KIT (Germany), NCSRD (Greece), CIEMAT (Spain), VUJE (Slovak Republic), SCK•CEN (Belgium) and Mutadis (France) in cooperation with the European platform NERIS. The course provided information on the AP, the scientific methods and tools developed for collecting information, analysing any nuclear or radiological event and providing information about the consequences and its future development. A particular attention was given to the conditions and means for pertinent, reliable and trustworthy information to be made available to the public in due time and according to its needs in the course of nuclear emergency and post-emergency context.

The main objective of the course was to train participants as regards the newly developed tools for further active participation in exercises, and using the AP as a focal point for collecting information, analysing any nuclear or radiological event and providing information about the consequences of the event and its future evolution. The course also covered the use of respectively traditional media and social media in the context of the Fukushima accident and trustworthiness of information and their reliability. 
The targeted audience of the workshop were researchers, organisations and actors in emergency and recovery preparedness and management from all levels and sectors.

The course consisted of lectures, demonstrations and practical sessions devoted to discussions and evaluation, and training and exercise sessions. These covered:

- the overview and applicability of the AP, technical realisation of AP, knowledge database of AP and the case-based reasoning, and 'Ask the expert' and web crawling;

- the operational use of the platform and practical sessions driven by the exercise scenario;

- the practical use of the AP and its applicability in future as improved means for the interaction of technical experts with authorities in charge and improved means for the communication from and to the public: traditional media and social media in the context of the Fukushima accident and trustworthiness of information and their reliability.

For the practical working sessions accompanying the lectures, a common scenario has been developed and used during the second day of the training course.

The theoretical and practical aspects of AP management and use have been addressed through:

- a half-day exercise in the form of table-top exercise and facilitated workshops split into two parts: the first part focused on information gathering and distributing, and the second part devoted to a nuclear event using INEX 5 exercise scenario; feedback from the exercise;

- round table discussion on the AP: use of scientific means, operational procedures, technical platform, information structuring and collection, virtual meeting room.

Each course day was closed with round table and discussion on the topic(s) of day or tools presented/ demonstrated and trained. The facilitated workshop conducted during the last day of workshop provided a possibility to widely discuss a number of issues among which:

- conditions to make platform useful for experts and the public;

- trustworthiness of the platform for experts, for public;

- interaction between users and the openness of information; and

- governance and maintenance of the platform.

The facilitated discussion revealed that the AP seems more useful for experts, although some outputs are useful for decision-makers. The AP can be used by experts, who are key communicators with the public, to pass knowledge and information. It could also have an active role for the media.

To ensure trustworthiness of the platform for experts and the public, several issues came up in the discussion: the quality control of the source of information; the previous experience of experts; the transparency, reliability and confidentiality of information exchanged; the dissemination; the competence and openness of experts and expert analysis processes; and the status of the platform as an international tool. The platform was deemed a very useful training tool.

The course lectures, demonstrations and practical sessions were very well received by the participants. The participants expressed the wish to continue the development of the AP beyond PREPARE project.

Future training course should cover more practical sessions, working directly with the system and its operating, more training in addition to facilitated workshops and discussions.

\section{Conclusions}

Education and training remains essential for the creation and maintenance of critical capacities in the field of emergency preparedness, response and recovery. The organisation of such courses in the framework of international projects and with cooperation from European radiation protection technical platforms (NERIS, ALLIANCE, EURADOS, MELODI), international organisations (IAEA, ICRP, UNSCEAR, European Commission and others) and communities experiencing the management of accidental or post-accidental exposure situations is highly beneficial. This ensures proper update of courses' content with the state-of-the-art theoretical knowledge, practice and decision-aid tools; incorporation of lessons learned from real events; and harmonisation of E\&T at European level. Future European projects should include basic and targeted courses addressing different aspects of emergency preparedness, response and recovery among their E\&T activities, notably with further integration of the feedback experience from the management of the consequences of the Fukushima accident. In addition, the results of the on-going research projects (among them OPERRA and COMET) would contribute to improve the training courses and allow the dissemination of the research activities.

Acknowledgement. The work leading to these results has received funding from the European Atomic Energy Community Seventh Framework Programme FP7/2012-2013 under grant agreement 323287. 